\title{
Butterflies (Lepidoptera: Papilionoidea and Hesperioidea) of the Private Reserve of Natural Heritage Fazenda Lontra/Saudade, Itanagra, Northern Coast of Bahia, Brazil
}

\author{
Márlon Paluch ${ }^{1,3}$, Olaf Hermann Hendrik Mielke ${ }^{2}$, Lucilio Matos Linhares ${ }^{I}$ \& Diego Carvalho da Silva ${ }^{I}$ \\ ${ }^{1}$ Universidade Federal do Recôncavo da Bahia, Centro de Ciências Agrárias, Ambientais e Biológicas, \\ Setor de Ciências Biológicas, CEP 44380-000, Cruz das Almas, Bahia, Brazil. \\ ${ }^{2}$ Universidade Federal do Paraná, Centro Politécnico, Departamento de Zoologia, CEP 81531-980, \\ Curitiba, Paraná, Brazil. \\ ${ }^{3}$ Corresponding author: Márlon Paluch, e-mail: marlonpaluch@gmail.com
}

PALUCH, M., MIELKE, O.H.H., LINHARES, L.M., SILVA, D.C. Butterflies (Lepidoptera: Papilionoidea and Hesperioidea) of the Private Reserve of Natural Heritage Fazenda Lontra/Saudade, Itanagra, Northern Coast of Bahia, Brazil. Biota Neotropica. 16(1): e20140085. http://dx.doi.org/10.1590/ 1676-0611-BN-2014-0085

\begin{abstract}
The Private Reserve of Natural Heritage Fazenda Lontra/Saudade (FLS), located in the Northern Coast of Bahia state, Brazil, is the largest (1,377.33 ha) preserved Atlantic Forest area between Paraguaçu and São Francisco rivers in Bahia and Sergipe, respectively. A list of 260 species belonging to six families of butterflies is presented herein, being recorded 4 species of Papilionidae, 16 of Pieridae, 29 of Lycaenidae, 41 of Riodinidae, 87 of Nymphalidae and 83 of Hesperiidae. The butterfly community was composed mainly by widespread species commonly found in open habitats. There were also many species typical of forested areas, as such Morpho telemachus richardus Fruhstorfer, 1898 (Nymphalidae: Morphini), a new record to the Northeastern Brazil.
\end{abstract}

Keywords: Inventory, Conservation, Species richness, Atlantic Forest.

PALUCH, M., MIELKE, O.H.H., LINHARES, L.M., SILVA, D.C. As borboletas (Lepidoptera: Papilionoidea e Hesperioidea) da Reserva Particular do Patrimônio Natural Fazenda Lontra/Saudade, Itanagra, Litoral Norte da Bahia, Brasil. Biota Neotropica. 16(1): e20140085. http://dx.doi.org/10.1590/ 1676-0611-BN-2014-0085

Resumo: A Reserva Particular do Patrimônio Natural Fazenda Lontra/Saudade (FLS), localizada no litoral norte do estado Bahia, Brasil, corresponde a maior área $(1.377,33$ ha) preservada de Mata Atlântica entre os rios Paraguaçu (Bahia) e São Francisco (Sergipe). Uma lista com 260 espécies de borboletas pertencentes a seis famílias é apresentada, sendo 4 espécies de Papilionidae, 16 de Pieridae, 29 de Lycaenidae, 41 de Riodinidae, 87 de Nymphalidae e 83 de Hesperiidae. A fauna foi composta principalmente por espécies de áreas abertas com ampla distribuição geográfica. Espécies típicas de áreas florestadas também estão presentes, uma delas, Morpho telemachus richardus Fruhstorfer, 1898 (Nymphalidae: Morphini), é um registro novo para a fauna de borboletas do nordeste do Brasil.

Palavras-chave: Inventário, Conservação, Riqueza de espécies, Mata Atlântica.

\section{Introduction}

The butterfly fauna of Northeastern Brazil was considered recently as largely unknown (Santos et al. 2008, Freitas \& Marini-Filho 2011). However, important areas of the biome Caatinga have been studied in the last years, mainly in Pernambuco (PE) and Bahia (BA). The Catimbau National Park (PE) (Nobre et al. 2008); the Ecological Park João Vasconcelos Sobrinho (PE), "Brejo de Altitude" enclave of Atlantic Forest inserted in the semi-arid (Paluch et al. 2011); northern portion of the "Chapada Diamantina" (BA) (Zacca \& Bravo 2012) and the "Serra da Fumaça" (BA) (Lima \& Zacca 2014), together represent a significant inventory of butterflies for the Brazilian semi-arid region.

To Atlantic Forest of Northeastern Brazil, recently was published a list of 140 species of butterflies of the "Serra da
Jibóia", a mountainous complex in the "Recôncavo" region of Bahia (Zacca et al. 2011). But, more significant data for this biome are quite old and were obtained during more than five years of collecting by Cardoso (1949), who listed 218 species of butterflies in urban areas of Maceió, Alagoas (AL). Additionally, Kesselring \& Ebert (1982) surveyed the urban fragment "Mata do Buraquinho" in João Pessoa, Paraíba (PB), and recorded 291 species. In this context, the present paper provides the first list of butterflies recorded in Itanagra, Northern Coast of Bahia, Brazil. It is expected that this list may offer subsidies for future conservation strategies in the region.

\section{Material and methods}

The study was conducted in the Private Reserve of Natural Heritage (Portuguese acronym, RPPN) "Fazenda Lontra/ 
Saudade" (FLS), Itanagra, located at the Northern Coast of Bahia ( $38^{\circ} 0.00^{\prime} \mathrm{W}$ and $\left.12^{\circ} 15.00^{\prime} \mathrm{S}\right)$. According Birdlife International (2014) FLS is the largest particular protected area of Atlantic Forest with extension of 1,377.33 ha, located between the Paraguaçu (BA) and São Francisco (SE) rivers, with possibility of reconnection with others forest fragments of the region. In this area, 183 species of plants from 56 families already were recorded, mostly of Myrtaceae, Fabaceae, Rubiaceae, Melastomataceae, Mimosaceae, Sapotaceae and Flacourtiaceae. The local economy is based on forestry (pine and eucalypt), subsistence agriculture and extensive cattle. The region has undulating relief, and is drained by a complex network of aquifers. The average annual rainfall is $1,800 \mathrm{~mm}$. The FLS is an example of environmental protection in the region, representing one of the most significant remnants of Atlantic Forest of Northern Coast of Bahia.

Butterflies were sampled between January to December 2012 (dry and wet seasons), totaling 12 field trips. Fieldwork was conducted from 9:00 to 15:00 h, during two days per month, with a total sampling effort of 288 hours. The samplings were carried out by two collectors along all habitats of the occidental side FLS.

The collected material was deposited in the entomological collections of the following institutions brazilian: Universidade Federal do Recôncavo da Bahia, Cruz das Almas (Laboratório de Sistemática e Conservação de Insetos - LASCI) and Departamento de Zoologia, Universidade Federal do Paraná, Curitiba (DZUP). Nomenclature follows Mielke (2005) (Hesperioidea) and Lamas (2004) (Papilionoidea), except for the genus Morpho Fabricius, 1807 (Morphini) according to Blandin (2007), and suprageneric categories of Nymphalidae modified after Wahlberg et al. (2009).

\section{Results and discussion}

A total of 260 butterfly species were recorded in the FLS. Of these, $177(68 \%)$ are Papilionoidea and $83(32 \%)$ Hesperioidea, with the following distribution: Papilionidae (4 spp., 1,5\%), Pieridae (16 spp., 6\%), Lycaenidae (29 spp., 11\%), Riodinidae (41 spp., 16\%), Nymphalidae (87 spp., $33,5 \%$ ), and Hesperiidae (83 spp., 32\% of the total) (Appendix 1). The butterfly fauna is composed by species commonly found in open areas and widely distributed in Brazil, and species more frequent in humid forests as well.

Four species of Papilionidae were recorded, and three of them were already expected for the area, Heraclides anchisiades capys (Hübner, [1809]), Heraclides thoas brasiliensis (Rothschild \& Jordan, 1906) and Battus polydamas polydamas (Linnaeus, 1758) because they are very common and occur throughout the Brazilian coast, Caatinga, and also in the "Brejos de Altitude" (Tyler et al. 1994, Nobre et al. 2008, Paluch et al. 2011, Zacca \& Bravo 2012). Parides zacynthus (Fabricius, 1793) has been considered restricted to the coastal plains, including "restingas" and lowland forests areas (Tyler et al. 1994). However, the subspecies $P$. zacynthus polymetus (Godart, 1819) was recently recorded in the Ecological Park João Vasconcelos Sobrinho, PE, about $100 \mathrm{~km}$ far from the coast (Paluch et al. 2011). In Bahia, this subspecies has been recorded to Salvador (Zacca 2009), but it was also collected in $15 \mathrm{~km}$ east of Lençóis, Bahia, about 300 $\mathrm{km}$ west from Salvador.

All sixteen species of Pieridae collected in FLS already were recorded in previous studies performed in Northeastern Brazil, and most of them are typically founded in open secondary forests, grasslands and more disturbed areas (Brown 1992). Only Leucidia elvina (Godart, 1819) (Coliadinae) and Enantia lina versicolora (Fruhstorfer, 1912) (Dismorphiinae) were collected in transects inside the forest.

Lycaenidae totaled 29 species in the FLS, same richness recorded for the Atlantic Forest of Maceió (AL) by Cardoso (1949). Kesselring \& Ebert (1982) recorded 46 species in João Pessoa, in a forest fragment along the Atlantic coastline. However, data were gathered during more than five years of observations.

The Riodinidae richness of FLS was surprising similar to the number of species found by Kesselring \& Ebert (1982). The presence of two sympatric subspecies of Stalachtis phlegia (Cramer, 1779), Stalachtis phlegia phlegetontia (Perty, 1833) and Stalachtis phlegia susanna (Fabricius, 1787) reveals an unusual taxonomic situation. Some authors consider Stalachtis susanna (Fabricius, 1787) as a valid species, for example Brown \& Freitas (2000b). Both taxa are very common, and were observed in all months flying at the same time in FLS. This situation can be best elucidated by a review on the status of all five subspecies (Lamas 2004).

Nymphalidae and Hesperiidae were the richest families, with similar numbers reported in other important forest areas in Northeastern Brazil (Cardoso 1949, Kesselring \& Ebert 1982). In general, Nymphalidae was represented by species widely distributed in different Brazilian biomes (from Amazonian borders to South Brazil).

Danaini (Danainae) is represented by two widespread species, previously mentioned for Bahia by Zacca et al. (2011) in the Atlantic Forest and Zacca \& Bravo (2012), Lima \& Zacca (2014) in the biome Caatinga. Ithomiini (Danainae) is represented by 7 species, same number recorded by Kesselring \& Ebert (1982), and includes common species which may be found throughout the year, for example, Mechanitis lysimnia nesaea Hübner, [1820] and Mechanitis polymnia casabranca Haensch, 1905.

Haeterini and Satyrini (Satyrinae) represent 23 species; the other inventoried areas in Northeastern Brazil have on average 13 species. The Neotropical genus Pierella Westwood, 1851, so far, with four species in northeastern Brazil in the states Alagoas, Paraíba, Bahia and Pernambuco (Cardoso 1949; Kesselring \& Ebert 1982; Zacca 2009; Paluch et al. 2011; Zacca et al. 2011), has revealed new taxa to the Northeastern Atlantic Forest, as Pierella sp. nov. in FLS described in Zacca et al. (in press) (Taxonomic revision of the "Pierella lamia species group"), as well as, Pierella lena atlantica Paluch, Zacca \& Siewert, 2015 in Paluch et al. (2015).

Brassolini (Satyrinae) is represented by five common and widespread species and Morphini (Satyrinae) by three species. Morpho helenor bahiana Fruhstorfer, 1897 (Blandin 2007) was recently mentioned by Zacca et al. (2011) as Morpho helenor achillaena (Hübner, 1823]) from Serra da Jiboia. The record of Morpho telemachus richardus Fruhstorfer, 1898 in FLS was important to enlarge $700 \mathrm{~km}$ to the east in the geographical distribution of this subspecies. The first and only known record is from southeastern Brazil, Minas Gerais, Teófilo Otoni. Seven mature larvae were collected in March on its hostplant Abuta sp. (Menispermaceae), a rare plant in the region. The genus Abuta Aubl. is also known as hostplant of Morpho telemachus martini Niepelt, 1933 in San Martín, Peru (Gallusser et al. 2010). In a greenhouse, five specimens of M. telemachus richardus emerged in April. 
Anaeini (Charaxinae) is represented by five species, Hypna clytemnestra forbesi Godman \& Salvin, 1884 is a typical subspecies of the Caatinga biome (Nobre et al., 2008), however, also known in the Atlantic Forest remnants of the "Recôncavo" and southern coast of Bahia (Zacca 2009; M. Paluch, pers. obs.).

Biblidinae and Cyrestinae are represented by 16 species, the tribes Coeini, Kallimini and Melitaeini (Nymphalinae) are represented by three, four and two common and widespread species, respectively. Anartia amathea amathea (Linnaeus, 1758) (Kallimini) generally restricted in rainforests of Northeastern region (Cardoso 1949; Kesselring \& Ebert 1982; Paluch et al. 2011), while Anartia jatrophae jatrophae (Linnaeus, 1763) is common in dry Caatinga, and also humid Atlantic Forest (Nobre et al. 2008; Zacca 2009; Zacca et al. 2011; Zacca \& Bravo 2012).

Limenitidinae is represented by four species: Adelpha cytherea cytherea (Linnaeus, 1758), also reported in Maceió, Alagoas (Cardoso 1949) and seems to be restricted to the Atlantic Forest biome, while Adelpha cytherea aea (C. Felder \& R. Felder, 1867) to the Semiarid region (Paluch et al. 2011, Zacca \& Bravo 2012); Adelpha leucates iphicleola Fruhstorfer, 1915 was recently recorded in two areas of Caatinga: Catimbau National Park and the northern portion of the Chapada Diamantina (Nobre et al. 2008, Zacca \& Bravo 2012). Adelpha melona melona (Hewitson, 1847) and Adelpha plesaure plesaure Hübner, 1823 are known from the Northeastern Atlantic Forest in Brazil (Cardoso 1949, Kesselring \& Ebert 1982, Zacca et al. 2011).

Heliconiinae is represented by thirteen taxa widely distributed in all extension of the Atlantic Forest. The northern border of the distribution of Heliconius ethilla narcaea Godart, 1819 (Heliconiini) seems to be Maceió, Alagoas Cardoso (1949); this subspecies was also collected at the "Serra da Jiboia" (Zacca et al. 2011), northern part of the "Chapada Diamantina" and at FLS. Heliconius ethilla flavomaculatus Weymer, 1894 is endemic to Pernambuco and Paraiba. Heliconius numata ethra (Hübner, [1831]) was re-collected in Northeastern Brazil, after 63 years since its unique record to Maceió, Alagoas (Cardoso 1949). However, this subspecies was cited by Brown \& Freitas (2000b) in Santa Teresa, Espírito Santo, near to the southern limits of Bahia. Actinote pellenea pellenea Hübner, [1821] (Acraeini) was previously recorded in southern Bahia (Jitaúna, Itanhém and Itamaraju); its northern subspecies Actinote pellenea aulodea Oberthür, 1917 is distributed in the Atlantic Forest of Alagoas, Pernambuco and Paraiba (Cardoso 1949, Paluch et al. 2011, Kesselring \& Ebert 1982).

Hesperiidae is considered the dominant family of butterflies in most well sampled Brazilian sites (C. Mielke 1994, Brown 2005, Morais et al. 2007, Francini et al. 2011, Dolibaina et al. 2011). In FLS skippers represented $32 \%$ of all butterflies species, with 83 recorded species. FLS corresponds to the second largest richness of Hesperiidae in the Northeastern Brazil, close to the 110 species recorded by Kesselring \& Ebert (1982).

Future studies can greatly enhance the richness of butterflies of the FLS, with sampling on the eastern side of the reserve, using "The Ahrenholz Technique", to attract species of skippers, Nymphalidae, Riodinidae and Pieridae (Lamas et al. 1993), together with "Van Someren-Rydon" traps, to capture others species of frugivorous butterflies, mainly of the subfamilies Satyrinae, Charaxinae and Biblidinae.

The FLS may be considered very important because it is one of the largest and best preserved remnants of Atlantic Forest in northeastern Brazil, this region is part of a biome with more than $95 \%$ of its original vegetation destroyed by human activities (Galindo-Leal \& Câmara 2003, Tabarelli et al. 2005). Considering
Nymphalidae as a surrogate $(25-29 \%)$ of the total butterfly fauna (Brown \& Freitas 2000a), the total richness in the FLS could reach 300 to 350 species, equivalent or higher to the total richness of "Mata do Buraquinho", João Pessoa, Paraiba, with a sampling effort of five years (Kesselring \& Ebert 1982). Based on the present data and other inventories performed in this area, FLS should be considered as a priority area for biodiversity conservation in the Atlantic Forest. Among the priority actions for the FLS, ecological monitoring and management of the surrounding environments should be included, so the area can keep sustaining health forests.

Appendix I. Butterflies (Papilionoidea and Hesperioidea) from the Private Reserve of Natural Heritage Fazenda Lontra/Saudade, Itanagra, Northern Coast, Bahia, Brazil. Number of species are provided within parenthesis for higher taxa. Species recorded in other areas of Atlantic Forest Northeastern: $1=$ Maceió, AL (Cardoso 1942); 2 = Mata do Buraquinho, João Pessoa, PB (Kesselring \& Ebert 1982); 3 = Ecological Park João Vasconcelos Sobrinho (Enclave of Atlantic Forest), Caruaru, PE (Paluch et al. 2011); 4 = Serra da Jibóia, Santa Terezinha, BA (Zacca et al. 2011).

\section{PAPILIONOIDEA (177)}

\section{Papilionidae (4)}

Papilioninae (4)

Troidini (2)

Battus polydamas polydamas (Linnaeus, 1758), 1,2,3,4

Parides zacynthus polymetus (Godart, 1819) ${ }^{2,3}$

Papilionini (2)

Heraclides anchisiades capys (Hübner, [1809]) $)^{1,2,3}$ $1906)^{1,2,3,4}$

Heraclides thoas brasiliensis (Rothschild \& Jordan,

\section{Pieridae (16)}

Dismorphiinae (1)

Enantia lina versicolora (Fruhstorfer, 1912) ${ }^{3}$

Coliadinae (12) Anteos clorinde (Godart, [1824]) $)^{1,2,3,4}$

Aphrissa statira statira (Cramer, 1777) $)^{1,3}$

Eurema agave pallida (Chavannes, 1850) ${ }^{3}$

Eurema albula albula (Cramer, 1775) $)^{1,3,4}$

Eurema elathea flavescens (Chavannes, 1850) ${ }^{3,4}$

Eurema phiale paula (Röber, 1909) (3, $4^{2}$

Leucidia elvina (Godart, 1819) $1,3,4$

Phoebis argante argante (Fabricius, 1775) $)^{1,2,4}$

Phoebis philea philea (Linnaeus, 1763) (1,2,3,4 $^{3,4}$

Phoebis sennae marcellina (Cramer, 1777) 3,4

Pyrisitia nise tenella (Boisduval, 1836) ${ }^{1,2,3,4}$

Rhabdodryas trite banksi (Breyer, 1939)

Pierinae (3)

Ascia monuste orseis (Godart, 1819) 1,2,3,4

Glutophrissa drusilla drusilla (Cramer, 1777) ${ }^{1,4}$

Itaballia demophile nimietes (Fruhstorfer, 1907) ${ }^{3}$

Lycaenidae (29)

Polyommatinae (3)

Hemiargus hanno hanno (Stoll, 1790) 2,3,4

Leptotes cassius cassius (Cramer, 1775) $)^{3,4}$

Zizula cyna (W.H. Edwards, 1881) $)^{2,3}$

Theclinae (26)

Arawacus ellida (Hewitson, 1867) ${ }^{2}$

Calycopis caulonia (Hewitson, 1877) ${ }^{3}$

Calycopis vesulus (Stoll, 1781)

Celmia celmus (Cramer, 1775) ${ }^{2}$ 
Evenus satyroides (Hewitson, 1865) ${ }^{1}$

Evenus sp.

Gargina gnosia (Hewitson, 1868)

Lamprospilus badaca (Hewitson, 1868) ${ }^{4}$

Ministrymon una (Hewitson, 1873) ${ }^{1}$

Ostrinotes tympania (Hewitson, 1869)

Panthiades hebraeus (Hewitson, 1867) ${ }^{2}$

Panthiades phaleros (Linnaeus, 1767) ${ }^{1,2}$

Pseudolycaena marsyas (Linnaeus, 1758) $)^{1,2}$

Rekoa meton (Cramer, 1779)

Rekoa palegon (Cramer, 1780) ${ }^{2,3,4}$

Strephonota ambrax (Westwood, 1852) ${ }^{1}$

Strephonota tephraeus (Geyer, 1837) ${ }^{2}$

Strymon bazochii (Godart, [1824]) 2,4

Strymon mulucha (Hewitson, 1867) 1,2,3,4

Theclopsis gargara (Hewitson, 1868)

Theclopsis sp.

Theritas hemon (Cramer, 1775) ${ }^{1,2,3,4}$

Theritas lisus (Stoll, 1790)

Theritas triquetra (Hewitson, 1865) $)^{1,2,4}$

Tmolus echion (Linnaeus, 1767) ${ }^{1,2}$

Ziegleria syllis (Godman \& Salvin, 1887) ${ }^{3}$

\section{Riodinidae (41)}

Euselasiinae (1)

Euselasiini (1)

Euselasia opalescens opalescens (Hewitson, [1855])

Riodininae (40)

Mesosemiini (7)

Hyphilaria parthenis (Westwood, 1851)

Leucochimona icare matatha (Hewitson, 1873) ${ }^{1,4}$

Mesosemia hesperina Buttler, 1874

Mesosemia methion methion Hewitson, 1860

Mesosemia nyctea lato Stichel, 1910

Perophthalma tullius (Fabricius, 1787) ${ }^{2,3}$

Semomesia geminus (Fabricius, 1793)

Eurybiini (2)

Eurybia halimede halimede (Hübner, [1807]) ${ }^{4}$

Eurybia molochina hyacinthina Stichel, 1910

Riodinini (10)

Baeotis euprepes H.W. Bates, 1868

Calephelis braziliensis McAlpine, 19712,3

Cyrenia martia androgyne Stichel, 1910

Isapis agyrtus sestus (Stichel, 1909) ${ }^{1}$

Melanis smithiae smithiae (Westwood, 1851) $)^{1,2,3}$

Metacharis ptolomaeus (Fabricius, 1793)

Panara jarbas jarbas (Drury, 1782) ${ }^{2}$

Rhetus periander (Cramer, 1777)

Syrmatia nyx (Hübner, [1817])

Themone pais pais (Hübner, [1820])

Symmachiini (6)

Mesene florus (Fabricius, 1793) ${ }^{2}$

Mesene phareus (Cramer, 1777) ${ }^{1,2}$

Mesene philonis Hewitson, 1874

Panaropsis inaria (Westwood, 1851) ${ }^{1}$

Pirascca sagaris satnius (Dalman, 1823) ${ }^{1,2,4}$

Symmachia menetas menetas (Drury, 1782)

Helicopini (1)

Anteros formosus (Cramer, 1777) ${ }^{2}$

Nymphidiini (8)

Calospila lucianus lucianus (Fabricius, 1793) $)^{1,2}$

Calospila parthaon (Dalman, 1823)
Juditha caucana (Stichel, 1911)

Nymphidium acherois erymanthus (Ménétriés, 1855)

Nymphidium lisimon attenuatum Stichel, 1929

Synargis calyce (C. Felder \& R. Felder, 1862) ${ }^{2,3}$

Synargis gela (Hewitson, [1853]) ${ }^{1,2}$

Synargis paulistina (Stichel, 1910)

Stalachtini (2)

Stalachtis phlegia phlegetontia (Perty, 1833)

Stalachtis phlegia susanna (Fabricius, 1787)

Incertae sedis (4)

Astraeodes areuta (Westwood, 1851) $)^{1,2}$

Calydna hiria (Godart, [1824])

Calydna sturnula (Geyer, 1837)

Emesis fatimella fatimella Westwood, $1851^{1}$

Nymphalidae (87)

Danainae (9)

Danaini (2)

Danaus eresimus plexaure (Godart, 1819) ${ }^{3}$

Lycorea halia discreta Haensch, 1909 3 ,4

Ithomiini (7)

Hypothyris euclea laphria (Doubleday, 1847) ${ }^{1,2,4}$

Hypothyris ninonia daetina (Weymer, 1899) ${ }^{1,2,3}$

Ithomia agnosia zikani d'Almeida, $1940^{4}$

Mechanitis lysimnia nesaea Hübner, [1820 $]^{1,2,3}$

Mechanitis polymnia casabranca Haensch, 1905

Oleria astrea thiemei (Oberthür, 1879)

Scada reckia reckia (Hübner, [1808]) $)^{3,4}$

Satyrinae (31)

Morphini (3)

Antirrhea archaea Hübner, [1822]

Morpho helenor bahiana Fruhstorfer, $1897^{4}$

Morpho telemachus richardus Fruhstorfer, 1898

Brassolini (5)

Brassolis sophorae laurentii Stichel, 1925

Caligo idomeneus ariphron Fruhstorfer, 1910

Caligo illioneus illioneus (Cramer, 1775) ${ }^{2,3,4}$

Eryphanis automedon (Cramer, 1775) $)^{2,3}$

Opsiphanes quiteria meridionalis Staudinger, $1887^{2}$

Haeterini (4)

Haetera piera diaphana Lucas, 1857

Pierella sp. nov.

Pierella lena atlantica Paluch, Zacca \& Siewert, 2015

Pierella hyalinus (Gmelin, [1790])

Satyrini (19)

Caeruleuptychia brixius (Godart, [1824])

Chloreuptychia arnaca (Fabricius, 1776) ${ }^{3}$

Chloreuptychia chlorimene (Hübner, [1819]) ${ }^{1}$

Chloreuptychia herseis (Godart, [1824])

Cissia myncea (Cramer, 1780) ${ }^{3}$

Erichthodes antonina (C. Felder \& R. Felder, 1867) ${ }^{1}$

Godartiana sp.

Hermeuptychia hermes (Fabricius, 1775) $)^{1,2,4}$

Magneuptychia lea (Cramer, 1777)

Magneuptychia libye (Linnaeus, 1767) ${ }^{2,3}$

Pareuptychia ocirrhoe interjecta (d' Almeida, 1952) ${ }^{1,3}$

Paryphthimoides poltys (Prittwitz, 1865) ${ }^{3}$

Pharneuptychia romanina (Bryk, 1953)

Pharneuptychia sp.

Taygetis echo (Cramer, 1775) ${ }^{2}$

Taygetis laches laches (Fabricius, 1793) ${ }^{1,3}$

Yphthimoides affinis (Butler, 1867) ${ }^{3}$ 
Yphthimoides manasses (C. Felder \& R. Felder, 1867) ${ }^{1,3}$ Yphthimoides renata (Stoll, 1780)

Charaxinae (5)

Anaeini (5)

Fountainea glycerium cratais (Hewitson, 1874) ${ }^{2}$

Fountainea ryphea phidile (Geyer, 1837) ${ }^{3}$

Hypna clytemnestra forbesi Godman \& Salvin, 1884

Memphis acidalia victoria $(\mathrm{H}$. Druce, 1877)

Memphis moruus stheno (Prittwitz, 1865)

Biblidinae (14)

Biblis hyperia nectanabis (Fruhstorfer, 1909) ${ }^{4}$

Callicore astarte codomannus (Fabricius, 1781)

Dynamine agacles agacles (Dalman, 1823) 1,3,4

Dynamine athemon athemaena (Hübner, [1824]) ${ }^{3}$

Dynamine postverta postverta (Cramer, 1779)

Ectima thecla thecla (Fabricius, 1796) ${ }^{1,3}$

Hamadryas amphinome amphinome (Linnaeus, 1767) $1,3,4$

Hamadryas arete (Doubleday, 1847) 1,2,3

Hamadryas februa februa (Hübner, [1823] ${ }^{3}$

Hamadryas feronia feronia (Linnaeus, 1758) ${ }^{1,3}$

Mestra dorcas hypermestra Hübner, [1825] $]^{1,2,3,4}$

Myscelia orsis (Drury, 1782) 1,2,3

Nica flavilla flavilla (Godart, [1824]) 4

Pyrrhogyra neaerea ophni Butler, 1870

Cyrestinae (2)

Marpesia chiron marius (Cramer, 1779) $)^{3,4}$

Marpesia petreus petreus (Cramer, 1776)

Nymphalinae (9)

Coeini (3)

Colobura dirce dirce (Linnaeus, 1758) ${ }^{1,2,3}$

Historis acheronta acheronta (Fabricius, 1775) ${ }^{1,2}$

Historis odius dious Lamas, $1995^{3}$

Kallimini (4)

Anartia amathea amathea (Linnaeus, 1758) $)^{1,2,3}$

Anartia jatrophae jatrophae (Linnaeus, 1763) 1,2,3,4 $^{1,2}$

Junonia evarete evarete (Cramer, 1779) ${ }^{1,2,3,4}$

Siproeta stelenes meridionalis (Fruhstorfer, 1909) ${ }^{1,3,4}$

Melitaeini (2)

Ortilia ithra (W.F. Kirby, 1900) ${ }^{2,3}$

Tegosa claudina (Eschscholtz, 1821) $)^{3,4}$

Limenitidinae (4)

Adelpha cytherea cytherea (Linnaeus, 1758) ${ }^{1}$

Adelpha iphicleola leucates Fruhstorfer, 1915 ${ }^{1}$

Adelpha melona melona (Hewitson, 1847) 1,2,4

Adelpha plesaure plesaure Hübner, $1823^{1,4}$

Heliconiinae (13)

Argynnini (1)

Euptoieta hegesia meridiania Stichel, $1938^{3}$

Acraeini (1)

Actinote pellenea pellenea Hübner, [1821]

Heliconiini (11)

Agraulis vanillae maculosa (Stichel, [1908]) 2,3,4

Dione juno juno (Cramer, 1779) $)^{1,2,3,4}$

Dryadula phaetusa (Linnaeus, 1758) ${ }^{2,3}$

Dryas iulia alcionea (Cramer, 1779) ${ }^{2,3,4}$

Eueides aliphera (Godart, 1819) ${ }^{1,4}$

Eueides isabella dianasa (Hübner, [1806]) ${ }^{1,2,3,4}$

Heliconius erato phyllis (Fabricius, 1775) 1,2,3,4

Heliconius ethilla narcaea Godart, $1819^{4}$

Heliconius numata ethra (Hübner, [1831])

Heliconius sara apseudes (Hübner, [1813]) 2,3,4

Philaethria wernickei (Röber, 1906) ${ }^{1,4}$

\section{HESPERIOIDEA (83)}

Hesperiidae (83)

Eudaminae (25)

Aguna asander asander (Hewitson, 1867) $1,2,3,4$

Aguna aurunce aurunce (Hewitson, 1867)

Astraptes anaphus anaphus (Cramer, 1777) 1,2,3

Astraptes janeira (Schaus, 1902) ${ }^{1}$

Augiades crinisus (Cramer, 1780) ${ }^{2}$

Autochton neis (Geyer, 1832) 2,3

Autochton zarex (Hübner, 1818) 1,2,3,4

Chioides catillus catillus (Cramer, 1779) $)^{2,3}$

Drephalys oriander (Hewitson, 1867)

Entheus priassus pralina Evans, $1952^{1}$

Euriphellus euribates polygius (Latreille, [1824])

Phanus australis L. D. Miller, $1965^{3}$

Phanus marshalli (W.F. Kirby, 1880)

Phocides distans distans (Herrich-Schäffer, 1869)

Salatis salatis (Stoll, 1782) ${ }^{2}$

Typhedanus crameri McHenry, $1960^{1}$

Typhedanus undulatus (Hewitson, 1867) ${ }^{2}$

Udranomia kikkawai (A.G. Weeks, 1906) ${ }^{2}$

Udranomia orcinus (C. Felder \& R. Felder, 1867) ${ }^{2}$

Urbanus cindra Evans, 1952

Urbanus dorantes dorantes (Stoll, 1790) ) $^{1,2,3,4}$

Urbanus esta Evans, 1952

Urbanus proteus proteus (Linnaeus, 1758)

Urbanus simplicius (Stoll, 1790) 2,3,4

Urbanus virescens (Mabille, 1877) ${ }^{2,4}$

Pyrginae (24)

Achlyodes busirus rioja Evans, $1953^{1}$

Anastrus neaeris narva Evans, 1953

Antigonus erosus (Hübner, [1812] ${ }^{2}$

Camptopleura janthinus (Capronnier, 1874)

Chiomara basigutta (Plötz, 1884) ${ }^{1,2}$

Cogia calchas (Herrich-Schäffer, 1869) (2,3 $^{2,3}$

Gesta gesta (Herrich-Schäffer, 1863) ${ }^{2,4}$

Helias phalaenoides palpalis (Latreille, [1824]) ${ }^{3}$

Heliopetes alana (Reakirt, 1868) 3,4

Heliopetes arsalte (Linnaeus, 1758) 2,3,4

Heliopetes omrina (Butler, 1870) 3,4

Mylon ander ander Evans, 1953

Nisoniades macarius (Herrich-Schäffer, 1870) $2,3,4$

Ouleus fridericus riona Evans, 1953

Pachyneuria duidae duidae (Bell, 1932)

Pyrgus orcus (Stoll, 1780) ${ }^{2,3,4}$

Pyrgus veturius Plötz, $1884^{2}$

Pythonides herennius lusorius Mabille, 1891

Pythonides limaea (Hewitson, 1868) ${ }^{2}$

Quadrus sp.

Sostrata festiva (Erichson, [1849])

Telemiades antiope (Plötz, 1882)

Timochreon doria (Plötz, 1884) ${ }^{2}$

Xenophanes tryxus (Stoll, 1780) $)^{1,2}$

Hesperiinae (33)

Anthoptus insignis (Plötz, 1882)

Artines sp.

Callimormus corades (C. Felder, 1862) ${ }^{2}$

Cobalus virbius hersilia (Plötz, 1882)

Corticea corticea (Plötz, 1882) ${ }^{3}$

Cymaenes distigma (Plötz, 1882)

Cymaenes tripunctus theogenis (Capronnier, 1874) ${ }^{4}$

Hylephila phyleus phyleus (Drury, 1773) $)^{1,2,3,4}$ 
Justinia justinianus justinianus (Latreille, [1824]) 2,4

Mnaseas bicolor inca Bell, 1930

Mnasilus allubita (Butler, 1877) ${ }^{2,3}$

Morys compta compta (Butler, 1877)

Nyctelius nyctelius nyctelius (Latreille, [1824]) ${ }^{2,3}$

Onophas columbaria (Herrich-Schäffer, 1870) ${ }^{2}$

Orses cynisca (Swainson, 1821)

Panoquina hecebolus (Scudder, 1872)

Paracarystus menestries menestries (Latreille, [1824]) ${ }^{4}$

Perichares philetes adela (Hewitson, 1867) $)^{2,3}$

Phanes aletes (Geyer, 1832) ${ }^{2}$

Phanes almoda (Hewitson, 1866)

Pompeius pompeius (Latreille, [1824]) ${ }^{1,2}$

Radiatus bradus Mielke, 1968

Saliana morsa Evans, 1955

Saturnus reticulata meton (Mabille, 1891)

Sodalia sodalis (Butler, 1877)

Talides sergestus (Cramer, 1775) ${ }^{1,2}$

Vacerra bonfilius bonfilius (Latreille, [1824])

Vehilius stictomenes stictomenes (Butler, 1877) ${ }^{2,3}$

Vettius artona (Hewitson, 1868) $2,3,4$

Vettius fantasos (Cramer, 1780) ${ }^{1,2}$

Vettius lafrenaye lafrenaye (Latreille, [1824])

Vettius phyllus prona Evans, 1955 1,3

Zariaspes mys (Hübner, [1808]) ${ }^{2}$

Pyrrhopyginae (1)

Pyrrhopyge phidias (Linnaeus, 1758)

\section{Acknowledgements}

We thank Dr. André Victor Lucci Freitas (UNICAMP) for their help in the identification of the species of Satyrini and Dr. Diego Rodrigo Dolibaina (UFPR) with the species of Lycaenidae and Riodinidae. We thank MSc. Thamara Zacca (UFPR) for reading and suggestions in the last version of the manuscript. Copener Florestal LTDA, Alagoinhas, Bahia for the permit of the inventory in the RPPN Fazenda Lontra/Saudade. This publication is part of the RedLep "Rede Nacional de Pesquisa e Conservação de Lepidópteros” SISBIOTA-Brasil/CNPq (563332/2010-7).

\section{References}

BIRDLIFE INTERNATIONAL 2014 Important Bird Areas factsheet: Itanagra. Downloaded from http://www.birdlife.org on 11/03/2014.

BLANDIN, P. 2007. The Systematics of the Genus Morpho, Lepidoptera Nymphalidae. Hillside Books, Canterbury, 227 pp.

BROWN Jr., K.S. 1992. Borboletas da Serra do Japi: diversidade, hábitats, recursos alimentares e variação temporal. In: História Natural da Serra do Japi - Ecologia e preservação de uma área florestal no Sudeste do Brasil (L.P.C. Morellato, ed.). Campinas: Editora da Unicamp, p. 142-187.

BROWN Jr., K.S. 2005. Geologic, evolutionary and ecological bases of the diversification of Neotropical butterflies: implications for conservation. In: Tropical rainforests: Past, Present and Future (E. Bermingham, C.W. Dick \& C. Moritz, eds.). Chicago, University of Chicago Press, p. 166-201.

BROWN Jr., K.S. \& FREITAS, A.V.L. 2000a. Atlantic Forest butterflies: indicators for landscape conservation. Biotropica 32, 934-956.

BROWN Jr., K.S. \& FREITAS, A.V.L. 2000b. Diversidade de Lepidoptera em Santa Teresa, Espírito Santo. Boletim do Museu de Biologia Mello Leitão, Santa Teresa, 11-12:71-118.

CARDOSO, A. 1949. Lepidópteros de Alagoas. Revista de Entomologia 20(1/3):427-436, http://dx.doi.org/10.1590/S0101-81751994000300001.
DOLIBAINA, D.R., MIELKE, O.H.H. \& CASAGRANDE, M.M. 2011. Borboletas (Papilionoidea e Hesperioidea) de Guarapuava e arredores, Paraná, Brasil: um inventário com base em 63 anos de registros. Biota Neotrop. 11(1): http://www.biotaneotropica.org. br/v11n1/pt/abstract?inventory + bn00211012011.

FRANCINI, R.B., DUARTE, M., MIELKE, O.H.H., CALDAS, A. \& FREITAS, A.V.L. 2011. Butterflies (Lepidoptera, Papilionoidea and Hesperioidea) of the "Baixada Santista" region, coastal São Paulo, Southeastern Brazil. Revista Brasileira de Entomologia 55: $55-68$.

FREITAS, A.V.L. \& MARINI-FILHO, O.J. 2011. Plano de Ação Nacional para Conservação dos Lepidópteros Ameaçados de Extinção. ICMBio, Brasília, Brazil, 124 pp.

GALINDO-LEAL, C. \& CÂMARA, I.G. 2003. Atlantic forest hotspots status: an overview. In: The Atlantic Forest of South America: biodiversity status, threats, and outlook (C. Galindo-Leal \& I.G. Câmara, eds.). Center for Applied Biodiversity Science e Island Press, Washington, D.C, p. 3-11.

GALLUSER, S., RAMIREZ, S.C. \& BLANDIN, P. 2010. Observaciones sobre el desarollo y polimorfismo de Morpho (Iphimedeia) telemachus (Linnaeus, 1758) en el noreste Peruano (Lepidoptera, Nymphalidae, Morphinae). Bulletin de la Société entomologique de France 115(1):5-15.

KESSELRING, J. \& EBERT, H. 1982. Relação das borboletas encontradas na "Mata do Buraquinho", João Pessoa, estado da Paraíba, Brasil. Revista Nordestina de Biologia 2 (1):105-108.

LAMAS, G. (ed.). 2004. Checklist: Part 4A Hesperioidea - Papilionoidea. In: Atlas of Neotropical Lepidoptera (J.B. Heppner, ed.) Association of Tropical Lepidoptera, Gainesville, 5A: XXXV + 439 p, http://dx.doi.org/10.1590/S1676-06032011000400020.

LAMAS, G., MIELKE, O.H.H. \& ROBBINS, R.K. 1993. The Ahrenholz technique for attracting tropical skippers (Hesperiidae). Journal of the Lepidopterists' Society 47(1):80-82.

LIMA, J.N.R. \& ZACCA, T. 2014. Lista de Espécies de Borboletas (Lepidoptera: Hesperioidea e Papilionoidea) de uma Área de Semiárido na Região Nordeste do Brasil. EntomoBrasilis 7(1):33-40, http://dx.doi.org/10.12741/ebrasilis.v7i1.351.

MIELKE, C.G.C. 1994. Papilionoidea e Hesperioidea (Lepidoptera) de Curitiba e seus arredores, Paraná, com notas taxonômicas sobre Hesperiidae. Revista Brasileira de Zoologia 11(4):759-776, http:// dx.doi.org/10.1590/S0101-81751994000400018.

MIELKE, O.H.H. 2005. Catalogue of the American Hesperioidea. 6vols. Sociedade Brasileira de Zoologia, Curitiba, $1536 \mathrm{pp}$.

MORAIS, A.B.B., ROMANOWSKI, H.P., ISERHARD, C.A., MARCHIORI, O. \& SEGUI, R. 2007. Mariposas del Sur de Sudamerica. Ciência \& Ambiente 35: 29-46.

NOBRE, C.E.B, SCHLINDWEIN, C. \& MIELKE, O.H.H. 2008. The butterflies (Lepidoptera: Papilionoidea and Hesperioidea) of the Catimbau National Park, Pernambuco, Brazil. Zootaxa 1751: 35-45.

PALUCH, M., MIELKE, O.H.H., NOBRE, C.E.B., CASAGRANDE, M.M., MELO, D.H.A. \& FREITAS, A.V.L. 2011. Butterflies (Lepidoptera: Papilionoidea and Hesperioidea) of the Parque Ecológico João Vasconcelos Sobrinho, Caruaru, Pernambuco, Brazil. Biota Neotrop. 11(4): http://www.biotaneotropica.org.br/v11n4/en/ abstract?inventory + bn01911042011.

PALUCH, M., ZACCA, T., SIEWERT, R.R., MIELKE, O.H.H. \& CASAGRANDE, M.M. 2015. A new subspecies of Pierella lena (Lepidoptera: Nymphalidae: Satyrinae) in northeastern Brazil. The Florida Entomologist 98(3):916-920, http://dx.doi.org/10.1653/024. 098.0316

SANTOS, E.C., MIELKE, O.H.H. \& CASAGRANDE, M.M. 2008. Inventários de borboletas no Brasil: Estado da arte e modelo de áreas prioritárias para pesquisa com vista à conservação. Natureza \& Conservação 6(2):68-90.

TABARELLI, M., PINTO, L.P., SILVA, M.C., HIROTA, M. \& BEDÊ, L. 2005. Challenges and opportunities for biodiversity conservation in the Brazilian Atlantic Forest. Conservation Biology 19: 695-700, http://dx.doi.org/10.1111/j.1523-1739.2005.00694.x. 
TYLER, H., BROWN JR, K.S. \& WILSON, K. 1994. Swallowtail butterflies of the Americas: a study in biological dynamics, ecological diversity, biosystematics and conservation. Scientific Publishers. Gainesville, Florida. 376p.

WAHLBERG, N., LENEVEU, J., KODANDARAMAIAH, U., PEÑA, C., NYLIN, S., FREITAS, A.V.L. \& BROWER, A.V.Z. 2009. Nymphalid butterflies diversify following near demise at the Cretaceous/Tertiary boundary. Proceedings of the Royal Society of London B 276:4295-4302, http://dx.doi.org/10.1098/rspb.2009.1303.

ZACCA, T. 2009. Espécies de borboletas (Lepidoptera: Papilionoidea e Hesperioidea) da Coleção Entomológica Prof. Johann Becker do Museu de Zoologia da Universidade Estadual de Feira de Santana, Bahia, Brasil. Sitientibus, Sér. Ciên. Biol. 9(2-3):165-173.
ZACCA, T., BRAVO, F. \& XAVIER, M.X. 2011. Butterflies (Lepidoptera: Papilionoidea and Hesperioidea) from Serra da Jibóia, Bahia State, Brazil. EntomoBrasilis 4(3):139-143, http://dx.doi.org/ 10.12741 .

ZACCA, T. \& BRAVO, F. 2012. Borboletas (Lepidoptera: Papilionoidea e Hesperioidea) da porção norte da Chapada Diamantina, Bahia, Brasil. Biota Neotrop. 12(2): http://www.biotaneotropica. org.br/v12n2/pt/abstract?inventory + bn00212022012.

ZACCA, T., SIEWERT, R.R., CASAGRANDE, M.M., MIELKE, O. H.H. \& PALUCH, M. Taxonomic revision of the "Pierella lamia species group" (Lepidoptera: Nymphalidae: Satyrinae) with descriptions of four new species from Brazil. Zootaxa (in press). 